\title{
THE STABILITY OF POWER SYSTEM MARKETS
}

\author{
Fernando Alvarado, Fellow \\ Department of Electrical and Computer Engineering \\ The University of Wisconsin - Madison \\ alvaradocengr . wisc.edu
}

\begin{abstract}
Market equilibrium conditions can be derived from more general dynamic equations describing the marketplace. Dynamic equations provide insights into the behavior and stability of markets which are not available from static models. For example, markets with a single supplier with declining linear costs (economies of scale) may or may not be stable, depending on specific cost characteristics. Markets with more than one supplier with declining linear costs are always unstable. This paper illustrates a situation where the removal of congestion makes a market unstable. Keywords: Pricing, economic stability, economies of scale, ISO.
\end{abstract}

\section{Introduction}

When designing Power Exchanges and policies for Independent System Operators for Electric Power Systems, it is necessary to consider whether the markets that underlie this mode of operation lead to wellbehaved (stable) equilibrium conditions. The potential instability of markets was recognized as a problem in the economics literature in the 1930s, where the use of static analysis techniques ("cobweb diagrams") was used to study the stability of markets [1]. Differential equation analysis of the stability of markets is described in $[2,3,4,5]$ and in many other economics references. This paper uses differential/algebraic equations and eigenvalue techniques to study power system markets. There has been little prior work in this area within the power systems literature, except for a less direct treatments of the problem in other contexts $[6,7]$.

Certain assumptions are made in the paper:

- Marginal production costs $\lambda_{g}$ are linear functions of the output quantity (the power $P_{g}$ ).

- Marginal benefit functions $\lambda_{d}$ are negatively-sloping linear functions of power consumption $\boldsymbol{P}_{\boldsymbol{d}}$.

- Any suppliers/consumers at a limit are considered as fixed injections/extractions to/from the market.

- Response of both suppliers and consumers to prices is not instantaneous. It is governed by first order single time constant differential equations.

PE-450-PWRS-0-05-1998 A paper recommended and approved by the IEEE Power System Analysis, Computing and Economics Committee of the IEEE Power Engineering Society for publication in the IEEE Transactions on Power Systems. Manuscript submitted August 7, 1997; made available for printing May 18, 1998.
- Except as otherwise noted, supply and demand are in balance at all times (no energy storage).

- Except as otherwise noted, there are no network constraints.

- Transportation (transmission) losses are negligible. Cases of increasing complexity are considered:

- A one-supplier one-consumer case.

- A fixed (inelastic) demand case.

- A case with $m$ suppliers and $n$ elastic consumers.

- The effect of network congestion on a market.

- The effect of congestion removal on market stability.

\section{The dynamics of simple economic systems}

If a supplier observes a market price $\lambda$ above his/her production cost $\lambda_{g i}$, it is assumed that the supplier will expand production until the marginal cost of production equals the price. The rate of expansion is proportional to the difference between the observed price and the actual production cost. The speed with which the generation power output $P_{g i}$ of supplier $i$ can respond is supplier dependent. It is denoted by a time constant $\tau_{g i}$ for supplier $i$. Let the price at any given time be $\lambda$. The above yields the following differential equation:

$$
\tau_{g i} \dot{P}_{g i}=\lambda-b_{g i}-c_{g i} P_{g i}
$$

where $b_{g i}+c_{g i} P_{g i}$ is the marginal cost $\lambda_{g i}$ of supplier $i$. A consumer demand $P_{d i}$ with a marginal benefit function $\lambda_{d i}$ above the marginal price will expand consumption until parity is attained. The speed of expansion is consumer dependent, and it is characterized by a time constant $\tau_{d i}$. The equation describing the behavior of a consumer is:

$$
\tau_{d i} \dot{P}_{d i}=b_{d i}+c_{d i} P_{d i}-\lambda
$$

where $b_{d i}+c_{d i} P_{d i}$ is the marginal consumer benefit.

The final condition required to characterize the marketplace is balance between supply and demand. If there is no energy storage, such a condition for the case of $m$ suppliers and $n$ consumers is characterized by:

$$
\sum_{i=1}^{m} P_{g i}=\sum_{i=1}^{n} P_{d i}
$$

or $P_{g}=P_{d}$ for the one-supplier one-consumer case.

Differential equations of this type can be derived, as it has been done here, under the assumption that the state variable of interest are the quantities (power generated and consumed, in this case). This corresponds to "Marshallian" formulation. Under these conditions, the price 
dynamics are a consequence of the quantity dynamics [3]. This model is appropriate when the quantities supplied and consumed are adjusted comparatively slowly relative to price. The dynamics of the model presented here include the further requirement that supply and demand be in precise balance at all times. An alternative dynamic model is possible, based on the use of prices rather than quantities as state variables. Such an approach has been called the "Walrasian" formulation. This alternative approach is not explored in this paper.

If a certain amount of energy can be stored in the system, the power balance equation 3 must be modified to include the dynamics of the stored energy. The case of energy imbalance, which in and of itself can lead to a market instability, is not considered in this paper, but is considered in [8].

\section{A one-supplier one-consumer case}

Consider the set of differential/algebraic equations (DAE) corresponding to the case of one supplier with demand elasticity $c_{g}$ and one consumer with demand elasticity $c_{d}$. The DAEs characterizing this case are:

$$
\begin{aligned}
\tau_{g} \dot{P}_{g} & =\lambda-b_{g}-c_{g} P_{g} \\
\tau_{d} \dot{P}_{d} & =b_{d}+c_{d} P_{d}-\lambda \\
P_{g} & =P_{d}
\end{aligned}
$$

which, in matrix form, can be expressed as:

$$
\left[\begin{array}{ccc}
\tau_{g} & 0 & 0 \\
0 & \tau_{d} & 0 \\
0 & 0 & 0
\end{array}\right]\left[\begin{array}{l}
\dot{P}_{g} \\
\dot{P}_{d} \\
\dot{\lambda}
\end{array}\right]=\left[\begin{array}{ccc}
-c_{g} & 0 & 1 \\
0 & +c_{d} & -1 \\
1 & -1 & 0
\end{array}\right]\left[\begin{array}{c}
P_{g} \\
P_{d} \\
\lambda
\end{array}\right]+\left[\begin{array}{c}
-b_{g} \\
b_{d} \\
0
\end{array}\right]
$$

The interpretation of these equations is as follows: the supplier acts in a way that tends to increase production when prices exceed production marginal costs. The consumer acts in a way that tends to increase consumption when marginal benefits exceed price.

The equilibrium for the DAE system above is obtained by setting the derivative terms to zero:

$$
\left[\begin{array}{ccr}
-c_{g} & 0 & 1 \\
0 & +c_{d} & -1 \\
1 & -1 & 0
\end{array}\right]\left[\begin{array}{c}
P_{g} \\
P_{d} \\
\lambda
\end{array}\right]=\left[\begin{array}{c}
b_{g} \\
-b_{d} \\
0
\end{array}\right]
$$

Solution of this algebraic linear problem leads to:

$$
\begin{aligned}
P_{g} & =\frac{b_{d}-b_{g}}{c_{g}-c_{d}} \\
P_{d} & =P_{g} \\
\lambda & =\frac{-b_{g} c_{d}+c_{g} b_{d}}{c_{g}-c_{d}}
\end{aligned}
$$

The following assumptions are reasonable:

- $c_{d}<0$, indicating that marginal consumer benefit decreases with consumption.

- $b_{d}>b_{g}$, indicating that initial consumer marginal benefit is greater than initial producer marginal cost.

- $b_{g}>0$ ("there is no free lunch").
- While normally $c_{g}>0$, the case of $c_{g} \leq 0$ cannot always be ruled out (economies of scale).

Under these assumptions:

- The solution occurs when the marginal cost of production equals the marginal benefit of corsumption.

- Equilibrium results in a positive price $\lambda>0$ as well as a positive amount of power $P_{g}=P_{d}>0$.

The price $\lambda$ can be eliminated from the original $d y-$ namic equations by adding equations 4 and 5 . This DAE model represents a first order system ${ }^{1}$. Substitution from 6, re-arrangement, and retention of the homogeneous portion of the result leads to:

$$
\left(\tau_{g}+\tau_{d}\right) \dot{P}_{g}=-\left(c_{g}-c_{d}\right) P_{g}
$$

The condition for the stability of this equation is that the eigenvalue associated with this problem be negative:

$$
\frac{c_{d}-c_{g}}{\tau_{g}+\tau_{d}}<0
$$

or simply $c_{g}>c_{d}$, since $\tau_{g}>0$ and $\tau_{d}>0$. Satisfying this condition is virtually assured, since $c_{d}<0$ (quite often $c_{d} \ll 0$ ). Thus, this type of market is stable, and static analysis is sufficient to ascertain the economic behavior of such a market.

The reason that so much effort has been taken to reach such an intuitive conclusion is because: arbitrarily complex cases can now be studied using the same eigenvalue method.

\section{The fixed-demand case}

The DAEs characterizing the two-supplier fixeddemand case are:

$$
\begin{aligned}
\tau_{g 1} \dot{P}_{g 1} & =\lambda-b_{g 1}-c_{g 1} P_{g 1} \\
\tau_{g 2} \dot{P}_{g 2} & =\lambda-b_{g 2}-c_{g 2} P_{g 2} \\
P_{g 1}+P_{g 2} & =P_{D}
\end{aligned}
$$

The equilibrium point is obtained from the solution of the following linear set of equations:

$$
\left[\begin{array}{ccc}
-c_{g 1} & 0 & 1 \\
0 & -c_{g 2} & 1 \\
1 & 1 & 0
\end{array}\right]\left[\begin{array}{c}
P_{g 1} \\
P_{g 2} \\
\lambda
\end{array}\right]=\left[\begin{array}{c}
b_{g 1} \\
b_{g 2} \\
-P_{D}
\end{array}\right]
$$

This solution generalizes readily to the case of $m$ suppliers and fixed demand:

$$
\left[\begin{array}{ccccc}
-c_{g 1} & 0 & \cdots & 0 & 1 \\
0 & -c_{g 2} & \cdots & 0 & 1 \\
\vdots & \vdots & \ddots & \vdots & \vdots \\
0 & 0 & \cdots & -c_{g m} & 1 \\
1 & 1 & \cdots & 1 & 0
\end{array}\right]\left[\begin{array}{c}
P_{g 1} \\
P_{g 2} \\
\vdots \\
P_{g m} \\
\lambda
\end{array}\right]=\left[\begin{array}{c}
b_{g 1} \\
b_{g 2} \\
\vdots \\
b_{g m} \\
P_{D}
\end{array}\right]
$$

The following are observations from the steady state equilibrium conditions:

\footnotetext{
${ }^{1}$ Implicit in this DAE model is the assumption that, although response of a market to a price signal is not instantaneous, such a response must result in power balance at all times.
} 
- Equilibrium occurs when all suppliers which are not constrained by either minimum or maximum limits operate at the same marginal cost $(\lambda)$.

- There are no a-priori assurances that the solution of equation 15 results in all suppliers with positive output at the equilibrium point ${ }^{2}$.

Eliminate (subtract) the first dynamic equation (equation 12) from the second (equation 13) and eliminate $P_{g 1}$ by substitution from equation 14 . The homogeneous part of the resulting differential equation is:

$$
\left(\tau_{g 1}+\tau_{g 2}\right) \dot{P}_{g 2}=-\left(c_{g 1}+c_{g 2}\right) P_{g 2}
$$

Observations from this equation are:

- Provided a solution exists, its stability is independent of the linear cost coefficients $b_{g 1}$ and $b_{g 2}$.

- If both $c_{g 1}>0$ and $c_{g 2}>0$, stability is assured.

- Because $\mathbf{T}$ is by construction a diagonal dominant allpositive matrix, it is sufficient to have $c_{g 1}+c_{g 2}>0$ to assure stability. Thus, it is possible for either $c_{1}$ or $c_{2}$ to be zero or slightly negative (but not both) and still have stable market conditions.

Generalization to the case of $m$ suppliers follows the same steps. The resulting homogeneous equations are:

$$
\begin{gathered}
{\left[\begin{array}{cccc}
\tau_{g 1}+\tau_{g 2} & \tau_{g 1} & \cdots & \tau_{g 1} \\
\tau_{g 1} & \tau_{g 1}+\tau_{g 3} & \cdots & \tau_{g 1} \\
\vdots & \vdots & \ddots & \vdots \\
\tau_{g 1} & \tau_{g 1} & \cdots & \tau_{g 1}+\tau_{g m}
\end{array}\right]\left[\begin{array}{c}
\dot{P}_{g 2} \\
\dot{P}_{g 3} \\
\vdots \\
\dot{P}_{g m}
\end{array}\right]=} \\
-\left[\begin{array}{ccccc}
c_{g 1}+c_{g 2} & c_{g 1} & \cdots & c_{g 1} \\
c_{g 1} & c_{g 1}+c_{g 3} & \cdots & c_{g 1} \\
\vdots & \vdots & \ddots & \vdots \\
c_{g 1} & c_{g 1} & \cdots & c_{g 1}+c_{g m}
\end{array}\right]\left[\begin{array}{c}
P_{g 2} \\
P_{g 3} \\
\vdots \\
P_{g m}
\end{array}\right]
\end{gathered}
$$

or, in more concise form:

$$
\tilde{\mathbf{T}} \dot{\tilde{\mathbf{P}}}=-\widetilde{\mathbf{C}} \tilde{\mathbf{P}}
$$

The dynamic characteristics of the market are dictated by the eigenvalues of this generalized eigenvalue problem. The following additional general observations can be made from the diagonal-dominance characteristics of these matrices:

- If $c_{g i}>0$ for all $i$, the result is a stable market (all eigenvalues are negative).

- If $c_{g i} \leq 0$ for two or more values of $i$, the result is necessarily an unstable market.

- Because $\widetilde{\mathbf{T}}$ is a diagonal-dominant positive matrix, it is sufficient to ascertain the stability properties of $\widetilde{\mathbf{C}}$ to establish the stability properties of $\widetilde{\mathbf{T}}^{-1} \widetilde{\mathbf{C}}$.

The practical consequence of the above is that markets can tolerate one supplier that exhibits economies of scale $\left(c_{i}<0\right)$, provided the economies are not too significant $^{3}$. It is, of course, unlikely that any one supply technology will exhibit indefinite economies of scale. However, the above can be used to help understand the

${ }^{2}$ Producers with negative output drop out of the market.

$$
{ }^{3} \text { If } c_{i}<0 \text {, then } \frac{1}{\left|c_{i}\right|}<\sum_{j=1, j \neq i}^{m} \frac{1}{\left|c_{j}\right|} \text {. }
$$

somewhat erratic behavior that power markets can be expected to exhibit over certain supply ranges. Negatively sloping marginal costs can be the result of:

- "Valve points"4 and other such conditions dictated by the efficiencies of various technologies.

- Requirements for minimum production levels.

- Startup and shutdown costs.

- Inherent economies of scale of certain technologies.

The erratic behavior that may result from these cost structures is a major factor in the determination of regulation ancillary service needs.

\section{The variable demand case}

The results of the previous two sections can be extended to the case where the dynamics of both supply and demand are taken into consideration. The general case of $m$ suppliers and $n$ consumers is considered.

The geıeral problem dynamic equations are:

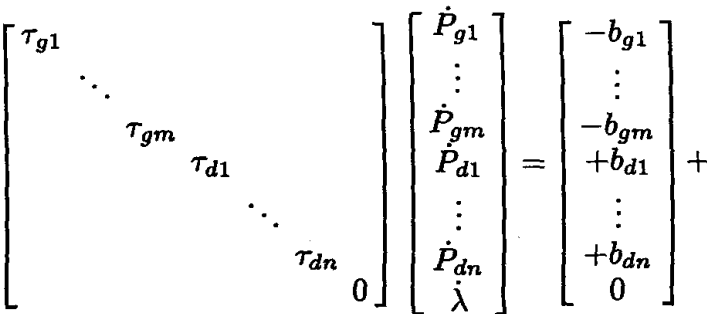

$$
\begin{aligned}
& {\left[\begin{array}{ccccccc}
-c_{g 1} & & & & & & 1 \\
& \ddots & & & & & \vdots \\
& & -c_{g m} & & & & 1 \\
& & & +c_{d 1} & & -1 \\
& & & & \ddots & & \vdots \\
& & & & & +c_{d n} & -1 \\
1 & \cdots & 1 & -1 & \cdots & -1 & 0
\end{array}\right]\left[\begin{array}{c}
P_{g 1} \\
\vdots \\
P_{g m} \\
P_{d 1} \\
\vdots \\
P_{d n} \\
\lambda
\end{array}\right]}
\end{aligned}
$$

The conditions for dynamic stability of the above problem can be obtained from the eigenvalues of equation 17 with:

$$
\begin{aligned}
\widetilde{\mathbf{T}} & =\left[\begin{array}{cccccc}
\tau_{g 1}+\tau_{g 2} & \cdots & \tau_{g 1} & -\tau_{g 1} & \cdots & -\tau_{g 1} \\
\vdots & \ddots & \vdots & \vdots & & \vdots \\
\tau_{g 1} & \tau_{g 1}+\tau_{g m} & -\tau_{g 1} & \cdots & -\tau_{g 1} \\
-\tau_{g 1} & \cdots & -\tau_{g 1} & \tau_{g 1}+\tau_{d 1} & \cdots & \tau_{g 1} \\
\vdots & & \vdots & \vdots & \ddots & \vdots \\
-\tau_{g 1} & \cdots & -\tau_{g 1} & \tau_{g 1} & \cdots \tau_{g 1}+\tau_{d n}
\end{array}\right] \\
\widetilde{\mathbf{C}}= & {\left[\begin{array}{cccccc}
-c_{g 1}-c_{g 2} & \cdots & -c_{g 1} & c_{g 1} & \cdots & c_{g 1} \\
\vdots & \ddots & \vdots & \vdots & & \vdots \\
-c_{g 1} & \cdots & -c_{g 1}-c_{g m} & c_{g 1} & \cdots & c_{g 1} \\
c_{g 1} & \cdots & c_{g 1} & -c_{g 1}+c_{d 1} \cdots & -c_{g 1} \\
\vdots & & \vdots & \vdots & \ddots & \vdots \\
c_{g 1} & \cdots & c_{g 1} & -c_{g 1} & \cdots & -c_{g 1}+c_{d n}
\end{array}\right] }
\end{aligned}
$$

${ }^{4}$ Valve points refer to situations when a partially open steam valve in a unit leads to inefficient operation. Many generating units have an incentive to operate near full throttle, as this results in lower marginal costs. 
which, for a two-supplier, one-load system, reduces to:

$$
\left[\begin{array}{lr}
\tau_{g 1}+\tau_{g 2} & -\tau_{g 1} \\
-\tau_{g 1} & \tau_{g 1}+\tau_{d 1}
\end{array}\right]\left[\begin{array}{l}
\dot{P}_{g 2} \\
\dot{P}_{d}
\end{array}\right]=\left[\begin{array}{rr}
-c_{g 1}-c_{g 2} & c_{g 1} \\
c_{g 1} & -c_{g 1}+c_{d}
\end{array}\right]\left[\begin{array}{l}
P_{g 2} \\
P_{d}
\end{array}\right]
$$

The eigenvalues of this problem dictate the stability of the market. If $c_{g i}>0$ for $1 \leq i \leq m$ and $c_{d i}>0$ for $1 \leq i \leq n$, stability is assured. This verifies that, even if demands are elastic, the market equilibrium is stable if no supplier exhibit economies of scale and no consumer exhibits "economies of consumption", that is, regions in their marginal benefit function where marginal benefits increase as consumption increases.

\section{The effect of congestion}

Congestion has the effect of imposing additional constraints on the market. In most markets, these effect are quite trivial and easy to visualize: the amounts of "goods" that can be shipped from A to B is limited, thus, the markets separate. In electric networks congestion is no less real, but it is harder to quantify and visualize because of the nature of network flows.

In the end, congestion restrictions can be characterized simply as one or more equality conditions that must be satisfied by the combination of all suppliers and consumers. Only "binding" congestion conditions are included as algebraic constraints and their Lagrange multipliers computed. Potential congestion is represented by inequalities that need to be monitored.

The characterization of congestion is done by means of a sensitivity matrix structure. Linear expressions relate individual power injections to individual flows. These equations are valuable because they characterize all viable trading thresholds which prevent a congested line from being further congested. This notion is the basis for the current coordinated trades proposal [9].

A single congested condition can be represented as a scalar additional equality constraint:

$$
\begin{aligned}
& S_{g 1} P_{g 1}+S_{g 2} P_{g 2} \cdots+S_{g m} P_{g m}+ \\
& \quad S_{d 1} P_{d 1}+S_{d 2} P_{d 2} \cdots+S_{d n} P_{d n}=s_{1}
\end{aligned}
$$

For the general case of $n_{s}$ congestion conditions the complete set of equality constraints is (the power balance conditions is always included):

$\left[\begin{array}{cccccc}1 & \cdots & 1 & -1 & \cdots & -1 \\ S_{g 11} & \cdots & S_{g 1 m} & S_{d 11} & \cdots & S_{d 1 n} \\ \vdots & & \vdots & \vdots & & \vdots \\ S_{g n_{s} 1} & \cdots & S_{g n_{\mathrm{s}} m} & S_{d n_{s} 1} & \cdots & S_{d n_{s} n}\end{array}\right]\left[\begin{array}{c}P_{g 1} \\ \vdots \\ P_{g m} \\ P_{d 1} \\ \vdots \\ P_{d n}\end{array}\right]=\left[\begin{array}{c}P_{D} \\ s_{1} \\ s_{2} \\ \vdots \\ s_{n_{s}}\end{array}\right]$

Congestion equality constraints are algebraic. Thus, analysis of the equilibrium as well as the stability properties of the congested market required the consideration of a DAE set of equations with multiple algebraic constraints. Of necessity, the congestion problem requires that higher-order nontrivial systems be considered. Simple one-supplier one-consumer examples are not meaningful in the presence of congestion. Furthermore, if the conditions for attaining feasibility are included, an equilibrium cannot be found because there are too many conditions specified. The issue is resolved by introducing shadow prices (Lagrange multipliers $\mu$ ) that play the role of congestion prices. If this is done, the complete dynamic equations for the congested $m$ supplier $n$-consumer case with $n_{s}$ active algebraic congestion conditions are:

$$
\left[\begin{array}{ll}
\mathbf{T} & \mathbf{0} \\
\mathbf{0} & \mathbf{0}
\end{array}\right]\left[\begin{array}{c}
\dot{\mathbf{P}} \\
\dot{\Lambda}
\end{array}\right]=\left[\begin{array}{cc}
\mathbf{C} & \mathbf{S}^{t} \\
\mathbf{S} & \mathbf{0}
\end{array}\right]\left[\begin{array}{c}
\mathbf{P} \\
\boldsymbol{\Lambda}
\end{array}\right]+\left[\begin{array}{c}
\mathbf{b} \\
\mathbf{s}
\end{array}\right]
$$

where:

$\mathbf{T}$ is a diagonal matrix with the time constants for all powers, generation as well as demand.

$\mathbf{P}$ is a vector of all powers ( $m$ generation powers and $n$ demand powers).

$\Lambda$ is a vector with $\lambda$ as its first entry and the Lagrange multipliers $\mu$ as the remaining entries.

$\mathbf{C}$ is the diagonal matrix of all quadratic cost coefficients $c_{g i}$ as well as $c_{d i}$.

$\mathbf{S}$ is the matrix corresponding to the sensitivities of the constraints. The first row is the power balance condition, thus its dimension is $m+n$ by $n_{s}+1$.

$\mathbf{b}$ is a vector of linear cost coefficients $-b_{g i}$ and $+b_{d i}$.

$\mathbf{s}$ is a vector with the value of the fixed demand in its first position and the values of the right hand sides in the constraint equations in the remaining positions.

The equilibrium point of these equations is obtained from the solution of the following algebraic equations:

$$
\left[\begin{array}{ll}
\mathbf{C} & \mathbf{S}^{t} \\
\mathbf{S} & 0
\end{array}\right]\left[\begin{array}{l}
\mathbf{P} \\
\boldsymbol{\Lambda}
\end{array}\right]=-\left[\begin{array}{l}
\mathbf{b} \\
\mathbf{S}
\end{array}\right]
$$

Key observations about these equations are:

- The main effect of congestion is a partial separation of markets: the solution no longer occurs at equal marginal costs for all producers [10].

- Which and how many equations join the algebraic constraints depends on the nature of congestion.

- The Lagrange multipliers $\mu$ are not unique. T.hey can all be altered by adding an arbitrary constant to all multipliers and the solution is unaltered.

Reduction of the DAE problem to a purely differential equation can be done by eliminating all the $n_{s}+1$ Lagrange multipliers, and replacing $n_{s}+1$ redundzint state variables in terms of a reduced set of $n+m-n_{s}-1$ non-redundant variables, obtaining in the end a set of $n+m-n_{s}-1$ purely differential equations. To do this, the equations above are organized as follows:

$$
\left[\begin{array}{ccc}
\mathbf{T}_{1} & 0 & 0 \\
0 & \mathbf{T}_{2} & 0 \\
0 & 0 & 0
\end{array}\right]\left[\begin{array}{c}
\dot{\mathbf{P}}_{1} \\
\dot{\mathbf{P}}_{2} \\
\dot{\Lambda}
\end{array}\right]=\left[\begin{array}{ccc}
\mathbf{C}_{1} & 0 & \mathbf{S}_{1}^{t} \\
0 & \mathbf{C}_{2} & \mathbf{S}_{2}^{t} \\
\mathbf{S}_{1} & \mathbf{S}_{2} & 0
\end{array}\right]\left[\begin{array}{c}
\mathbf{F}_{1} \\
\mathbf{F}_{2} \\
\Lambda
\end{array}\right]
$$

where $\mathbf{S}_{1}$ corresponds to a nonsingular $n_{s}+1$ by $n_{s}+1$ submatrix of $\mathbf{S}$, and $\mathbf{P}_{1}$ corresponds to a subset of $\mathbf{P}$ denoting redundant power variables. The classification 
of $\boldsymbol{P}$ into redundant and non-redundant states is irrelevant. It is subject only to the condition that $S_{1}$ be nonsingular. Reduction and elimination of $\Lambda$ and $\mathbf{P}_{1}$ yields the following reduced purely differential equations:

$$
\left[\mathbf{T}_{2}+\mathbf{S}_{2}^{t} \mathbf{S}_{1}^{-t} \mathbf{T}_{1} \mathbf{S}_{1}^{-1} \mathbf{S}_{2}\right] \dot{\mathbf{P}}_{2}=\left[\mathbf{C}_{2}+\mathbf{S}_{2}^{t} \mathbf{S}_{1}^{-t} \mathbf{C}_{1} \mathbf{S}_{1}^{-1} \mathbf{S}_{2}\right] \mathbf{P}_{2}
$$

where $\mathbf{S}_{1}^{-t}$ is the transposed inverse of $\mathbf{S}_{1}$.

The stability characteristics of the congested problem depend on the location of the eigenvalues for this reduced dynamic system. Observe that:

- The effect of congestion on the dynamics is to reduce the order of the dynamic interactions.

- The dynamics of the congestion pricing mechanism have not been considered.

Thus, congestion alters the number and nature of the already present algebraic constraints under which the market operates. The converse is also true: the removal of congestion can lead to a market instability.

\section{The numerical examples}

Table 1 illustrates a variety of parameters and conditions for the one-supplier one-consumer case. Observe the following:

- Changes in the linear cost coefficients $b$ affect $P_{g}$ and $P_{d}$. These changes also affect the cost $\lambda$.

- Changes to $b$ do not affect the eigenvalue.

- Modifications to $\tau_{g}$ and $\tau_{d}$ affect the dynamic characteristics, but not the operating point.

- Changes to $c_{g}$ and/or $c_{d}$ affect both.

Actual values for the time constants $\tau_{g}$ are likely to be dependent on the technology, on the status of the supplier at the time when the price signal is received, and on the means by which these signals are communicated to the supplier. For example, a hydro generating unit that is operating within a market where real time signaling is permitted may be able to respond to changes in price in a matter of seconds, whereas the time constant for a large thermal plant could be from several hours to more than a day. Likewise, the time constant for the demand depend on the technology at the disposal of the supplier, ranging from a near-instantaneous capability for certain forms of real time pricing proposals, to 24 hours or more for "day ahead" real time pricing.

Table 1: Equilibrium and dynamic characteristics of a one supplier one demand system without congestion. Changes relative to the base case are highlighted.

\begin{tabular}{|rrr|rrr|rrr|r|}
\hline \multicolumn{3}{|c|}{ Suppliers } & \multicolumn{2}{|c|}{ Consumers } & \multicolumn{3}{|c|}{ SS solution } & \\
\hline$\tau_{g}$ & $c_{g}$ & $b_{g}$ & $\tau_{d}$ & $c_{d}$ & $b_{d}$ & $\bar{P}_{g}$ & $\bar{P}_{d}$ & $\lambda$ & Eig. \\
\hline 0.3 & 0.5 & 2 & 0.2 & -0.5 & 10 & 8.0 & 8.0 & 6.00 & -2.00 \\
0.3 & 0.5 & 4 & 0.2 & -0.5 & $\mathbf{9}$ & 5.0 & 5.0 & 6.50 & -2.00 \\
0.2 & 0.5 & 2 & 0.2 & -0.5 & 10 & 8.0 & 8.0 & 6.00 & -2.50 \\
0.3 & 0.5 & 2 & 0.2 & -0.2 & 10 & 11.4 & 11.4 & 7.71 & -1.40 \\
0.3 & 0.5 & 2 & 0.2 & -2.0 & 10 & 3.2 & 3.2 & 3.60 & -5.00 \\
\hline
\end{tabular}

Table 2 illustrates a two-supplier inelastic demand example. It shows the values of the steady-state solution point as well as the dynamic characteristics of a market with inelastic demand. As before, only one eigenvalue is needed to characterize this case. The following observations can be made:
- If one supplier has economies of scale and the other does not, the issue of stability depends on the values of certain other parameters.

- If the market is stable for one set of values of $\tau$ (market delays), it is stable for any other value of $\tau$.

Table 2: Equilibrium and dynamic characteristics of a two-supplier fixed-demand system without congestion. Changes relative to the base case are highlighted.

\begin{tabular}{|c|c|c|c|c|c|}
\hline \multicolumn{4}{|c|}{ Input data } & Equilibrium & \\
\hline$\tau_{g}$ & $c_{g}$ & $b_{g}$ & $\overline{P_{D}}$ & $\begin{array}{ll}P_{g} & \lambda\end{array}$ & Eig. \\
\hline 0.3 & 0.5 & 2.0 & 10.0 & $\frac{y}{1.43}$ & -1.40 \\
\hline 0.2 & 0.2 & 1.0 & & 8.57 & \\
\hline 0.3 & 0.5 & 2.0 & 8.0 & 0.86 & -1.40 \\
\hline 0.2 & 0.2 & 1.0 & & 7.14 & \\
\hline$\overline{0.3}$ & 0.5 & 2.0 & 10.0 & 5.71 & -1.40 \\
\hline 0.2 & 0.2 & 4.0 & & 4.29 & \\
\hline 0.3 & 0.5 & 2.0 & 10.0 & 3.33 & -0.60 \\
\hline 0.2 & -0.2 & 5.0 & & 6.67 & \\
\hline 0.3 & -0.3 & 3.5 & $\overline{10.0}$ & 5.00 & 0.20 \\
\hline 0.2 & 0.2 & 1.0 & & 5.00 & \\
\hline
\end{tabular}

Changes in the elasticity coefficients directly affect the solution eigenvalues and can even make the market unstable. The last case has a positive eigenvalue.

Table 3 illustrates a set of multi-supplier multiconsumer examples. Interesting numbers are indicated boldface in the Table.

Table 3: Three-supplier two-consumer case for various time constants and cost coefficients. The last two cases exhibit economies of scale. The last case is unstable.

\begin{tabular}{|rrr|rrr|rr|r|r|}
\hline \multicolumn{3}{|c|}{ Suppliers } & \multicolumn{2}{|c|}{ Consumers } & \multicolumn{2}{|c|}{ SS solution } & \\
\hline$\tau_{g}$ & \multicolumn{1}{|c|}{$c_{g}$} & $b_{g}$ & $\tau_{d}$ & $c_{d}$ & $b_{d}$ & $P_{g}$ & $P_{d}$ & $\lambda$ & Eig. \\
\hline 0.3 & 0.5 & 2.0 & 0.20 & -0.50 & 10.0 & 2.52 & 13.48 & 3.26 & -1.24 \\
0.2 & 0.2 & 1.0 & 0.25 & -0.60 & 8.0 & 11.31 & 7.90 & & -1.85 \\
0.1 & 0.3 & 1.0 & & & & 7.54 & & & -2.74 \\
& & & & & & & & -2.44 \\
\hline 0.3 & 0.5 & 2.0 & 0.20 & -0.50 & 10.0 & 4.67 & 11.33 & 4.33 & -0.04 \\
0.2 & -0.1 & 4.5 & 0.25 & -0.60 & 8.0 & 1.67 & 6.11 & & -1.83 \\
0.1 & 0.3 & 1.0 & & & & 11.11 & & & -2.74 \\
& & & & & & & & & -2.44 \\
\hline 0.3 & 0.5 & 2.0 & 0.20 & -0.50 & 10.0 & 3.62 & 12.38 & 3.81 & 0.50 \\
0.2 & -0.1 & 5.0 & 0.25 & -0.60 & 8.0 & 11.92 & 6.99 & & -0.93 \\
0.1 & -0.05 & 4.0 & & & & 3.84 & & & -1.95 \\
& & & & & & & & -2.45 \\
\hline
\end{tabular}

These examples verify the earlier analysis that indicates that, if economies of scale exist, unstable markets can occur. The following inferences can be drawn:

- The steady state operating point depends on the linear cost coefficients $b$.

- The steady state operating point does not depend on the time constants $\tau$ or the quadratic cost coefficients.

- The stability of the operating point depends on the quadratic coefficients $c_{g}$ or $c_{d}$.

The next set of examples considers the effect of congestion on both the equilibrium point for the market and the stability of this equilibrium point. The congestion example is illustrated in Table 4. This table illustrates a three-supplier two-consumer case with one, two and three congestion constraints active. In addition to 
Table 4: A three-supplier two-consumer case with increasing degrees of congestion.

\begin{tabular}{|r|rrr|rrr|rr|r|r|}
\hline & \multicolumn{3}{|c|}{ Suppliers } & \multicolumn{3}{|c|}{ Consumers } & \multicolumn{3}{c|}{ SS solution } & \\
\hline$n_{s}$ & $\tau_{g}$ & $c_{g}$ & $b_{g}$ & $\tau_{d}$ & $c_{d}$ & $b_{d}$ & $P_{g}$ & $\bar{P}_{d}$ & $\lambda$ & Eig. \\
\hline 0 & 0.1 & 0.3 & 1 & 0.20 & -0.50 & 10 & 7.54 & 13.48 & 3.26 & -1.24 \\
& 0.3 & 0.5 & 2 & 0.25 & -0.60 & 8 & 2.52 & 7.90 & & -1.85 \\
& 0.2 & 0.2 & 1 & & & & 11.31 & & & -2.74 \\
& & & & & & & & & -2.44 \\
\hline 1 & 0.1 & 0.3 & 1 & 0.20 & -0.50 & 10 & 0.40 & 8.53 & 3.43 & -1.24 \\
& 0.3 & 0.5 & 2 & 0.25 & -0.60 & 8 & 7.47 & 11.47 & 23.07 & -2.03 \\
& 0.2 & 0.2 & 1 & & & & 12.13 & & & -2.58 \\
\hline 2 & 0.1 & 0.3 & 1 & 0.20 & -0.50 & 10 & 1.89 & 7.68 & 6.27 & -2.00 \\
& 0.3 & 0.5 & 2 & 0.25 & -0.60 & 8 & 11.52 & 8.05 & 14.96 & -2.42 \\
& 0.2 & 0.2 & 1 & & & & 2.31 & & 16.01 & \\
\hline 3 & 0.1 & 0.3 & 1.0 & 0.20 & -0.50 & 10.0 & 2.30 & 7.56 & 6.49 & -2.05 \\
& 0.3 & 0.5 & 2.0 & 0.25 & -0.60 & 8.0 & 11.51 & 8.35 & 13.51 & \\
& 0.2 & 0.2 & 1.0 & & & & 2.10 & & 16.51 & \\
& & & & & & & & 1.18 & \\
\hline
\end{tabular}

the usual power balance condition, the congestion constraints in this hypothetical example are characterized by the following conditions:

$$
\left[\begin{array}{rrrrr}
1 & 1 & 1 & -1 & -1 \\
0.1 & -0.1 & 0 & 0.1 & -0.1 \\
0.2 & 0 & 0.3 & -0.1 & -0.1 \\
0.1 & 0.1 & 0.1 & 0 & -0.4
\end{array}\right]\left[\begin{array}{l}
P_{g 1} \\
P_{g 2} \\
P_{g 3} \\
P_{d 1} \\
P_{d 2}
\end{array}\right]=\left[\begin{array}{c}
0 \\
1.00 \\
0.50 \\
1.75
\end{array}\right]
$$

Four cases of increasing congestion are considered. The first case $\left(n_{s}=0\right)$ considers only the first rows in the constraint equations 20 . The second case $\left(n_{b}=\right.$ 1) considers the first two rows. The next case $\left(n_{s}=\right.$ 2) considers three rows in equation 20). The last case $\left(n_{s}=3\right)$ considers all rows.

The following are observations from this case:

- As the number of active congestion constraints increases, the order of the system dynamics (the number of eigenvalues) decreases.

- Congestion affects the number of eigenvalues. However, if the case is stable without congestion, it remains stable after the onset of congestion.

- Congestion affects the operating point significantly.

- The operating cost increases as congestion increases.

- Although not illustrated in this example, it is possible for values of the Lagrange multiplier entries in $\Lambda$ to change sign. This is an indication that the corresponding constraint has become superseded (irrelevant) and must be removed.

Table 5: Illustration of one case where the removal of a constraint leads to a market instability.

\begin{tabular}{|r|rrr|rrr|rr|r|r|}
\hline & \multicolumn{3}{|c|}{ Suppliers } & \multicolumn{2}{|c|}{ Consumers } & \multicolumn{3}{|c|}{ SS solution } & \\
\hline$n_{s}$ & $\tau_{g}$ & $c_{g}$ & $b_{g}$ & $\tau_{d}$ & $c_{d}$ & $b_{d}$ & $P_{g}$ & $P_{d}$ & $\lambda$ & Eig. \\
\hline 1 & 0.1 & -0.05 & 3 & 0.2 & -0.5 & 10 & 13.95 & 16.44 & 2.67 & -1.05 \\
& 0.3 & -0.02 & 2 & 0.25 & -0.6 & 8 & 10.89 & 9.50 & 1.01 & -2.45 \\
& 0.2 & 0.5 & 1.5 & & & & 1.08 & & & -0.96 \\
\hline 0 & 0.1 & -0.05 & 3 & 0.2 & -0.5 & 10 & 21.55 & 16.16 & 1.92 & 0.19 \\
& 0.3 & -0.02 & 2 & 0.25 & -0.6 & 8 & 3.88 & 10.13 & & -1.01 \\
& 0.2 & 0.5 & 1.5 & & & & 0.84 & & & -2.43 \\
& & & & & & & & & -2.5 \\
\hline
\end{tabular}

The last example is perhaps the most startling. Consider now a case with three suppliers and two demands as illustrated in Table 5 . The case if first considered under the condition that there is congestion. One free flowing area consists of generator 2 and load 1 . The second area consists of generators 1 and 3 and load 2. As can be seen from the table, such a congestion is stable. However, if the congestion is removed and free trades are permitted among all parties, a market instability results. This potential difficulty can be managed provided appropriate trade and market rules are devised.

An instability implies that some generators will expand their output at an ever increasing rate while forcing others to suddenly reduce their output. They will eventually stop their expansion when either (a) they eventually reach an operating point where their marginal cost is no longer a declining function or (b) they reach their maximum output capability. The behavior occurs because the presumed equilibrium point is no longer a minimum cost point, but rather it becomes a saddle-node. Suppliers react accordingly, in an effort to attain economic efficiency. The difficulty implied by an instability is the sudden and discontinuous nature of the behavior. An instability in the context of this paper does not mean that the system fails or collapses ${ }^{5}$

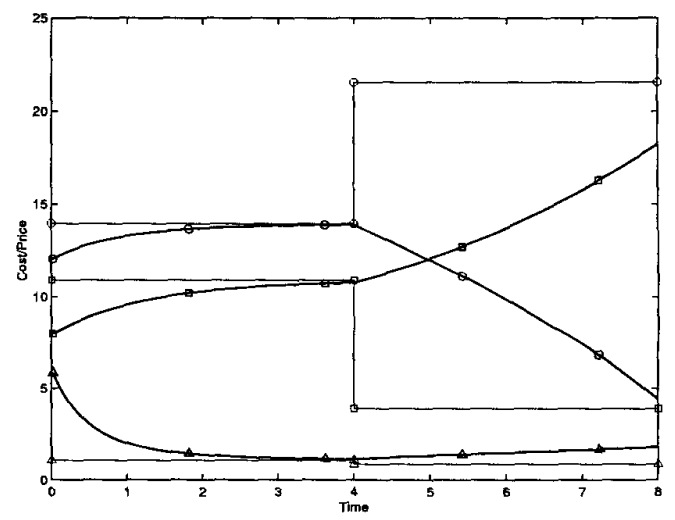

Fig. 1: All suppliers converge toward equilibrium. When congestion is removed at $t=4$ the suppliers do not converge to the new equilibrium. This figure corresponds to table 5 data.

Figure 1 is used to illustrate this last point. This figure shows the supplier outputs are shown for all three suppliers. Initially at $t=0$ there is congestion and the market is not at equilibrium (although the energy balance requirement is met). However, the market rapidly converges to steady-state values. At $t=4$ the congestion is removed. The system does not converge to its new equilibrium. Instead, it diverges until some limit is reached.

\footnotetext{
${ }^{5}$ However, discontinuous behavior that leads to drastic flow changes can indirectly lead to a reduction in system security as a result of the need for greater control action and the transients that these changes entail. It may also lead to reluctance to participate in the market due to increased economic risk.
} 


\section{Extensions}

The basic methodology for the understanding of electric power markets can be extended in a number of directions. A most important direction is the case where energy imbalance is permitted. A recent report [8] illustrates how the effect of energy imbalance can lead to an oscillatory instability (Hopf bifurcation) of the market. Likewise, the interaction of market delays and time constants with electro-mechanical delays and time constants can, under some circumstances, lead to unexpected market behavior.

\section{Conclusions}

Stable well-behaved markets for electric power require consideration of the dynamics of those markets. Ignoring the dynamics of markets for electric power can result in markets that are economically unstable exhibiting great price volatility. This, in turn, can lead to energy systems that are less secure with frequent outages and emergencies, as security constraints are not properly enforced in a timely fashion. It can also lead to conditions where monopolistic behavior becomes possible, even with multiple competing suppliers. This can be avoided by the proper design of the market and its parameters.

This paper has considered the specific case of a market instability that occurs as a result of the elimination of a constraint. Such an event would have a definite bearing on the decision(s) leading to system operation. These types of instability can in all probability be removed by the design of appropriate forward markets for electricity, but the role of congestion cannot be ignored in the design of these financial instruments.

\section{REFERENCES}

[1] M. Ezekiel. The cobweb theorem. Quarterly Journal of Economics, 52(2):255-278, 1938.

[2] D. Hawkins. Some conditions for macroeconomic stability. Econometrica, pages 310-322, October 1948.

[3] P. A. Samuelson. Foundations of Economic Analysis, Enlarged Edition. Harvard University Press, 1983.

[4] B. Beavis and I. M. Dobbs. Optimization and Stability Theory for Economic Analysis. Cambridge University Press, 1990.

[5] G. L. Thompson and S. A. O. Thore. Computational economics. In Annals of Operations Research, volume 68. Boltzer Science, 1997.

[6] R. Baldick, R. J. Kaye, and F. F. Wu. Electricity tariffs under imperfect knowledge of participant benefits. IEEE Transactions on Power Systems, 7(4):1471-1482, November 1992.

[7] J. Ruusunen, R. P. Hämäläinen, and M. Räsänen Game theoretic modelling in the dynamic pricing of electricity. In R. P. Hämäläinen and H. K. Ehtamo, editors, Lecture Notes in Control and Information, volume 157. Springer-Verlag, 1990.

[8] F. Alvarado. The dynamics of power system markets. Technical Report PSERC-97-01, Power Systems Engineering Research Consortium (PSERC), The University of Wisconsin, March 1997.

[9] F. F. Wu. Coordinated multilateral trades for electric power networks. In Power System Computation Conference, pages 786-792, August 1996.

[10] H. Glavitsch and F. L. Alvarado. Management of multiple congested conditions in unbundled operation of a power system. IEEE Transactions on Power Systems, 1997. (To appear.) Presented at PICA, Columbus, Ohio, May 1997.

Fernando L. Alvarado is a Professor of Electrical and Computer Engineering at the University of Wisconsin and a consultant to Christensen Associates. He received his $\mathrm{Ph} . \mathrm{D}$. from the University of Michigan in 1972. He has numerous publications on numerical methods and computer techniques for power systems application as well as topics on power system economic analysis. He is a Fellow of IEEE. 